\title{
Using magnetic resonance imaging in animal models to guide drug development in multiple sclerosis
}

\author{
Nabeela Nathoo',2, V Wee Yong ${ }^{1,3}$ and Jeff F Dunn ${ }^{1-4}$
}

\author{
'Hotchkiss Brain Institute, University of Calgary, Canada ${ }^{2}$ Department of Radiology, University of Calgary, Canada ${ }^{3}$ Department of Clinical \\ Neurosciences, University of Calgary, Canada \\ ${ }^{4}$ Experimental Imaging Centre, University of Calgary, Canada.
}

\begin{abstract}
Major advances are taking place in the development of therapeutics for multiple sclerosis (MS), with a move past traditional immunomodulatory/immunosuppressive therapies toward medications aimed at promoting remyelination or neuroprotection. With an increase in diversity of MS therapies comes the need to assess the effectiveness of such therapies. Magnetic resonance imaging (MRI) is one of the main tools used to evaluate the effectiveness of MS therapeutics in clinical trials. As all new therapeutics for MS are tested in animal models first, it is logical that MRI be incorporated into preclinical studies assessing therapeutics. Here, we review key papers showing how MR imaging has been combined with a range of animal models to evaluate potential therapeutics for MS. We also advise on how to maximize the potential for incorporating MRI into preclinical studies evaluating possible therapeutics for MS, which should improve the likelihood of discovering new medications for the condition.
\end{abstract}

Keywords: Magnetic resonance imaging, experimental autoimmune encephalomyelitis, Theiler's murine encephalomyelitis virus, cuprizone, lysolecithin, disease-modifying therapies

\section{Introduction}

There has been a substantial increase in potential therapeutics for multiple sclerosis (MS) at the preclinical stage, including anti-inflammatory, remyelinating and neuroprotective therapies. As such, there is a greater need for evaluating these potential therapies using a noninvasive method like magnetic resonance imaging (MRI). As MRI is frequently used as a primary outcome measure in phase II clinical trials and as a secondary outcome measure in phase III clinical trials, ${ }^{1}$ understanding what work has been performed using MRI in animal models of MS for assessing potential therapeutics is essential. Here, we review studies evaluating potential therapeutics in MS using animal models that incorporate MRI. We used PubMed to search for articles based on the search terms of the animal models discussed, MRI and treatment. We also reviewed citation lists from these papers. Because of space limitations, we did not include all papers found, but we believe we have captured key papers, and we use these to highlight key concepts. We will discuss how to improve the integration of MRI into studies assessing potential therapeutics in MS at the preclinical stage. Understanding what has been conducted in this area and what future steps can be taken may help guide drug development in MS.

\section{Overview of MRI methods that have been used to assess therapeutics for MS in animal models}

Various MRI methods have been used to assess the effects of potential therapeutics on disease processes inherent to MS at the preclinical stage. Rodents have historically been used to test the sensitivity of MR methods to detect pathologies such as blood-brain barrier (BBB) breakdown, inflammation, demyelination and white matter changes, and atrophy. Although detection thresholds may vary between humans and animals, in part because of the more variable pathologies in humans, imaging metrics that work in animals tend to work in humans.

Corresponding author:

Jeff F Dunn, Department of Radiology, University of Calgary, 3330

Hospital Drive, NW, Calgary, AB T2N 4NI, Canada.

Email:dunnj@ucalgary.ca 
BBB breakdown can be assessed using $\mathrm{T}_{1}$-weighted MRI with gadolinium (Gd). In instances of BBB breakdown, Gd enters the brain parenchyma, leading to enhancement or hyperintensity (brightness) on $\mathrm{T}_{1}$-weighted MRI.

Inflammation can be studied at the cellular level with MRI using iron oxide-based nanoparticle contrast agents, such as ultrasmall superparamagnetic iron oxide (USPIO) particles, which range in size from $10 \mathrm{~nm}$ to $50 \mathrm{~nm}$. As iron is paramagnetic, accumulation of iron nanoparticles in tissue leads to hypointense (dark) signals on $T_{2}$ and $T_{2}{ }^{*}$ weighted MRI or hyperintense (bright) signals on $\mathrm{T}_{1}$-weighted MRI. These nanoparticles can be used to label specific inflammatory cell populations. When delivered systemically, iron nanoparticles are taken up by monocytes circulating in the blood. ${ }^{2}$ Iron nanoparticles are approved for human use and have been used in research studies with MS patients to track monocytes, ${ }^{3,4}$ although these are currently being used off label. To the best of our knowledge, the only brand of iron nanoparticles approved by the United States (US) Food and Drug Administration (FDA) is ferumoxytol for iron therapy in chronic kidney disease. ${ }^{5}$

Magnetization transfer imaging (MTI) is based on the ability of hydrogen protons in macromolecules (e.g. lipids, proteins), which are restricted in motion, to exchange with hydrogen protons in free water. ${ }^{6}$ In instances where membrane lipid content is reduced, magnetization transfer ratio (MTR) is decreased, as the number of bound hydrogen protons available to exchange magnetization with hydrogen in free water is reduced. ${ }^{7}$

Diffusion tensor imaging (DTI) uses the diffusion of water in tissue to relay information about tissue microstructure. The diffusion of water can be equal in all directions, which is termed isotropic diffusion (as would be seen in cerebrospinal fluid), or it can be restricted in some directions because of the presence of barriers including axons and myelin, leading to anisotropic diffusion. Specific values, termed scalar measures, can be calculated from DTI datasets and have been shown to correlate with demyelination and axonal injury. ${ }^{8}$

Perfusion MRI can be used to provide measures of blood flow and blood volume. Dynamic contrast-enhanced imaging is one type of perfusion imaging that tracks the movement of a bolus tracer passing through a capillary network by acquiring multiple images following injection of the bolus. ${ }^{9}$

\section{Evaluating potential MS therapeutics in animal models using MRI}

\section{Experimental autoimmune encephalomyelitis (EAE)}

EAE is the animal model most frequently used to study MS. EAE is induced by stimulating the immune system through antigen presentation of components related to specific central nervous system (CNS) components, leading to an autoimmune disorder targeting the white matter.

The EAE model was a key component in the creation of three disease-modifying therapies (DMTs) for MS: glatiramer acetate, mitoxantrone and natalizumab. ${ }^{10}$ Most, if not all, therapeutic agents for MS are tested at a preclinical stage using EAE to determine if they are effective in the animal model before being applied to the patient population.

\section{Assessing the effectiveness of DMTs using MRI}

Within the realm of evaluating potential therapies for MS, MRI plays a significant role, with MRI metrics frequently being used as outcome measures for clinical trials. ${ }^{11}$ If a potential agent does not show benefit on MRI in phase II, it will not be pursued in a phase III trial. It seems logical, then, to use preclinical MRI studies as a screening tool for potential agents to determine if there is enough of a benefit provided by the agent to take it into clinical trials.

Some DMTs already being utilized as treatments for MS have been tested in EAE using MRI to assess the effectiveness of these agents. Glatiramer acetate is an antiinflammatory therapy for MS. When applied in EAE, mice treated with glatiramer acetate had reduced ventricle volume, increased MTR and reduced apparent diffusion coefficient compared to untreated EAE mice; these results corresponded to immunohistochemistry of reduced cell infiltration, demyelination and axonal changes in glatiramer acetate-treated EAE mice compared to untreated EAE mice. These alterations in MRI metrics are indicative of the anti-inflammatory and neuroprotective properties of glatiramer acetate. ${ }^{12}$ However, it is unknown whether the neuroprotective benefits offered by glatiramer acetate are a secondary result of being anti-inflammatory, or if there is a separate mechanism of action conferring neuroprotection. Glatiramer acetate has been shown to promote repair through various mechanisms including increased expression of growth factors and an elevation in the number of oligodendrocyte precursor cells. ${ }^{13}$

Interferon- $\beta$ is another immunomodulatory treatment for MS. One of the mechanisms of action of interferon- $\beta$ is to reduce leukocyte trafficking into the CNS at the level of the BBB that has been seen as reduction in Gd enhancement in MRI. ${ }^{14}$ The effects of interferon- $\beta$ have been tested in a focal EAE model (where the injection was made in the corpus callosum) to determine if it helps prevent damage associated with re-activation of a lesion following administration of interleukin (IL)-1 $\beta$, which is produced during peripheral infections, both in the brain and systemically. ${ }^{15}$ In this study, IL- $1 \beta$ was administered via an adenoviral vector, as the adenoviral vector would only be taken up by the liver, leading to IL-1 $\beta$ expression only in the periphery. Damage due to reactivation of the lesion following administration of the adenovirus containing IL- $1 \beta$ was reduced when interferon- $\beta$ was administered. Specifically, BBB breakdown was reduced (as shown by reduced Gd enhancement 


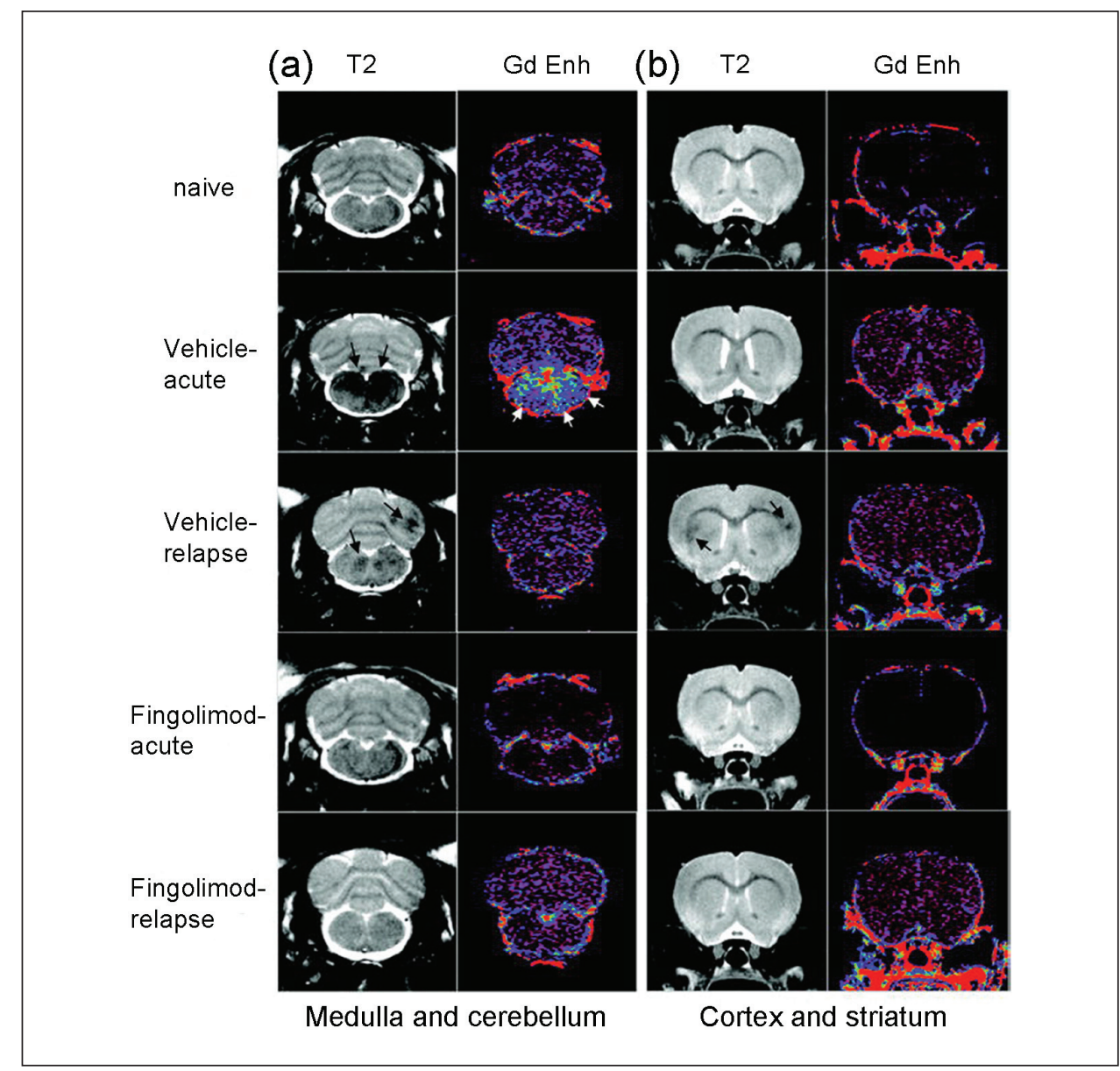

Figure I. Fingolimod treatment prevents development of USPIO and Gd lesions in EAE animals at acute disease and during relapse. $\mathrm{T}_{2}$-weighted images and signal enhancement $\mathrm{Gd}$ images are shown for naive, vehicle-acute, vehicle-relapse, fingolimod-acute and fingolimod-relapse animals. (a) shows images of the medulla and cerebellum. (b) shows images of the cortex and striatum. Black arrows and white arrows point out lesions on $\mathrm{T}_{2}$ or areas of $\mathrm{Gd}$ enhancement in vehicle-treated EAE animals that are not seen in the fingolimod-treated EAE animals. (Adapted from Rausch et al., 2004, Journal of Magnetic Resonance Imaging ${ }^{21}$ ).

USPIO: ultrasmall superparamagnetic iron oxide; Gd: gadolinium; EAE: experimental autoimmune encephalomyelitis.

in the ipsilateral meninges), apparent diffusion coefficient hyperintensity was reduced, and regional cerebral blood volume was reduced as measured using dynamic susceptibility contrast perfusion MRI. These MRI alterations were in agreement with reduced inflammation and demyelination as assessed using histology. ${ }^{16}$

Natalizumab is a humanized monoclonal antibody against very late antigen-4 (VLA-4), an integrin ( $\alpha 4$ integrin) expressed on leukocytes. Anti-VLA-4 attenuates EAE through preventing leukocyte adhesion onto the inflamed brain endothelium by blocking the interaction between VLA-4 and vascular cell adhesion molecule-1.17 In EAE, USPIOs were applied to track monocyte infiltration into the brains of animals treated with monoclonal anti-rat VLA-4 antibodies. Anti-VLA-4 did not completely prevent monocyte entry into the CNS, but it reduced USPIO-based $\mathrm{T}_{1}$ lesion volumes on MRI compared to vehicle antibody-treated animals. Furthermore, MRI volume changes correlated with histological staining for macrophages/microglia. ${ }^{18}$ Anti- $\alpha 4$ integrin has also been used in guinea pigs with acute EAE between 11 and 17 days post-immunization, where it was shown to reduce Gd enhancement in $\mathrm{T}_{1}$ - and edema in $\mathrm{T}_{2}$-weighted MRI in treated EAE animals compared with untreated EAE animals. ${ }^{19}$

Fingolimod (previously known as FTY720) is the first licensed oral treatment for MS that acts as a sphingosine-1 phosphate receptor modulator, leading to immunomodulatory effects. ${ }^{20}$ The first study using fingolimod in EAE with $\mathrm{MRI}^{21}$ took place approximately two years before results from phase II clinical trials were published, which makes this an interesting example of a preclinical MRI study that may have guided subsequent clinical trials. There were no Gd or USPIO lesions in rats that received fingolimod during the acute phase of EAE, whereas untreated EAE rats had Gd-enhancing lesions that also accumulated USPIOs (Figure 1). These results correlated well with scoring for disability and with histology for 


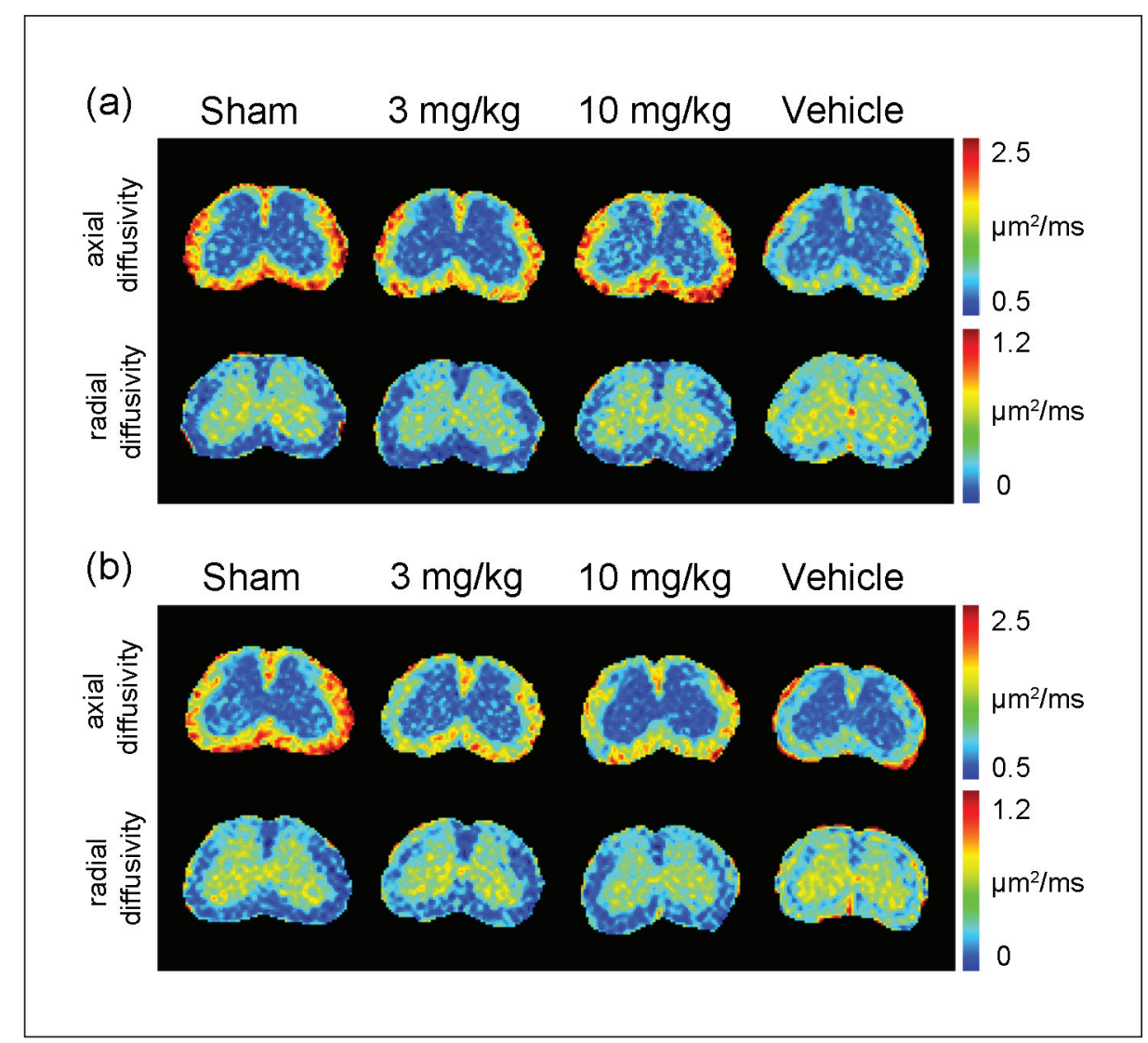

Figure 2. Axial diffusivity is elevated and radial diffusivity is reduced in fingolimod-treated EAE animals compared to vehicle-treated EAE mice. DTI parameter maps at the L4 level of the spinal cord are shown for animals treated at the time of EAE induction (a) or at EAE disease onset (b). Axial diffusivity is higher and radial diffusivity is lower in the white matter of EAE mice treated with 3 $\mathrm{mg} / \mathrm{kg}$ or $10 \mathrm{mg} / \mathrm{kg}$ fingolimod compared to vehicle-treated EAE mice, suggesting a protective effect with fingolimod on these DTI parameters. (Adapted from Wang et al., 20I3, NMR in Biomedicine ${ }^{22}$ ).

EAE: experimental autoimmune encephalomyelitis; DTI: diffusion tensor imaging.

macrophages. ${ }^{21}$ Fingolimod has also been assessed in EAE using DTI where it was observed that axial diffusivity was elevated and radial diffusivity was reduced in EAE mice treated with fingolimod compared to those treated with vehicle, regardless of whether treatment was administered at disease induction or at disease onset (Figure 2). These alterations in axial diffusivity and radial diffusivity are in agreement with histological findings, which showed greater preservation of axons and myelin in fingolimod-treated EAE mice compared to vehicle-treated EAE mice. These results suggest that fingolimod has a protective effect in EAE.22 So far, results gleaned from MRI studies in EAE using established MS therapies have corroborated human clinical data.

\section{Assessing the effectiveness of other potential therapeutics using MRI}

Combining imaging with treatment studies has resulted in encouraging breakthroughs. A promising new agent that promotes remyelination in animal models, anti-leucine-rich repeat and immunoglobulin domain-containing
Nogo receptor-interacting protein 1 (anti-LINGO-1), is an immunoglobulin $\mathrm{G}$ ( $\mathrm{IgG}$ ) antibody that binds to LINGO-1 that is currently in phase I clinical trials for MS. The effects of anti-LINGO-1 have been studied in EAE using DTI. It was observed that EAE animals treated with anti-LINGO-1 showed significantly increased fractional anisotropy compared to EAE animals treated with an IgG control antibody. This was seen both when antiLINGO-1 was administered before clinical symptoms presented and when given post-clinical presentation in EAE. Fractional anisotropy values were correlated with toluidine blue staining for myelin, suggesting that antiLINGO-1 promotes remyelination in EAE (Figure 3). ${ }^{23}$ However, it is interesting that only fractional anisotropy was used in this study, as it is a measure of tissue integrity, and is not necessarily specific to extent of myelination. It would be interesting if other DTI scalar measures were studied, such as radial diffusivity, which has been proposed to be a more specific marker of myelin status. ${ }^{24}$

Minocycline is an inhibitor of microglial activation and attenuates apoptosis,,$^{25}$ and is currently in a phase III clinical trial in early MS (ClinicalTrials.gov Identifier: 


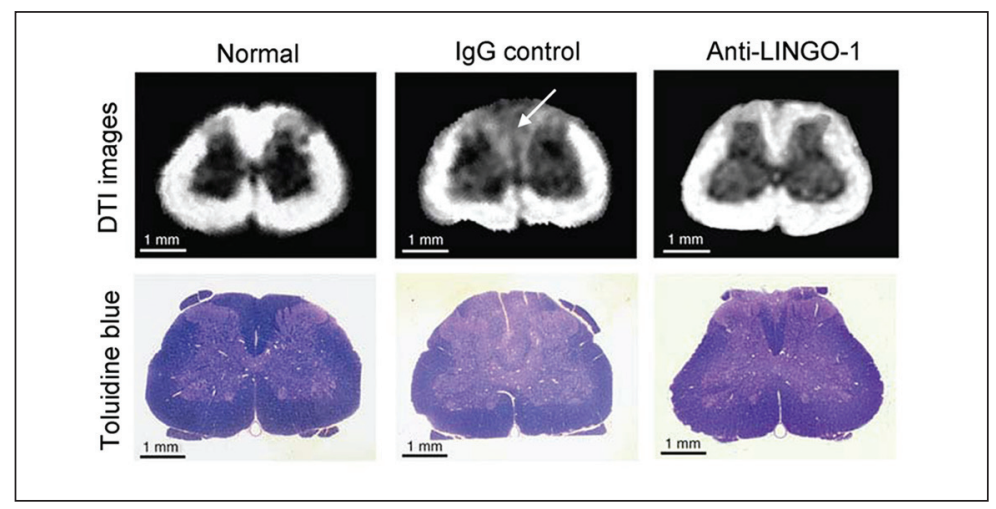

Figure 3. Anti-LINGO-I-treated EAE rats have higher fractional anisotropy values in the dorsal area of the lumbar spinal cord compared to IgG control-treated EAE rats. DTI images from normal rats, IgG control-treated EAE rats, and anti-LINGO-I-treated EAE rats are shown with corresponding toluidine blue-stained spinal cord sections. Low fractional anisotropy values (darker areas; white arrow) are seen in the dorsal column of the IgG control-treated EAE rats, which correlated with a loss of toluidine blue staining, indicative of demyelination. Fractional anisotropy values were much higher in anti-LINGO-I-treated EAE rats and in normal rats, which corresponded to greater myelination seen with toluidine blue staining. (Adapted from Mi et al., 2007, Nature Medicine ${ }^{23}$ ). anti-LINGO-I: anti-leucine-rich repeat and Ig domain-containing Nogo receptor-interacting protein I; EAE: experimental autoimmune encephalomyelitis; IgG: immunoglobulin G; DTI: diffusion tensor imaging.

NCT00666887). A study combined the administration of minocycline with prednisone in EAE, with minocycline being administered at disease onset until the end of the study, while prednisone was administered on days $1-7$ of disease and then at relapse for seven days, after which it was withdrawn. This combination treatment reduced EAE disease severity, prevented the development of $T_{2}$ lesions and localized Gd enhancement on $T_{1}$ to the meninges. These effects were seen up to 60 days after induction of disease. ${ }^{26}$

Dietary modifications have also been tested in the EAE model. The ketogenic diet, a diet high in fat, normal in protein and low in carbohydrates, reduces inflammation ${ }^{27}$ and has been shown to reduce production of reactive oxygen species. ${ }^{28}$ There was reduced $T_{2}$ lesion volume and preservation of hippocampal volumes in EAE mice fed the ketogenic diet as compared to EAE mice fed a standard diet. ${ }^{29}$

There is renewed interest in the use of adrenocorticotropic hormone (ACTH) to treat acute relapses in MS with the knowledge that it exerts anti-inflammatory and immunomodulatory effects through melanocortin pathways. ${ }^{30} \mathrm{An}$ $\mathrm{ACTH}$ analog $(\mathrm{ACTH})_{4-9}$, was applied to EAE rats at the time of EAE induction for 21 weeks, when the experiment ended. EAE rats treated with the analog showed similar $\mathrm{T}_{2}$ lesion distributions compared to untreated EAE rats in the periventricular white matter and hippocampus, but treated rats had reductions in lesion volume by more than $80 \%$. Regions with high $\mathrm{T}_{2}$ signals coincided with areas of inflammation, demyelination and edema. ${ }^{31}$

Hyperbaric oxygen therapy (HBOT) has also been tested as a potential therapy in the EAE model, where no differences were observed in any characteristics of lesions, including their volumes, locations and relaxation times as studied using quantitative $T_{1}$ and $T_{2}$, even though clinical symptoms in EAE were delayed. ${ }^{32}$ These results are in agreement with a recent meta-analysis of the use of HBOT in MS which concluded that HBOT did not appear to have any significant benefit. ${ }^{33}$

\section{Theiler's murine encephalomyelitis virus (TMEV)}

TMEV is a model of MS that is a gastrointestinal pathogen belonging to the RNA picornavirus family. When TMEV is administered intracerebrally in susceptible mouse strains, it can produce demyelination. ${ }^{34,35}$

\section{Assessing the effectiveness of treatment using MRI}

A recombinant form of a human monoclonal IgM antibody, rHIgM22, has been shown to promote remyelination independent of immunomodulation in the TMEV model. ${ }^{36,37}$ This agent was applied as a single dose in mice with TMEV for six months, where $\mathrm{T}_{2}$-weighted MRI was performed prior to initiation of treatment and five weeks after treatment. Five weeks after treatment, $\mathrm{T}_{2}$ lesion load and lesion volumes were significantly reduced in rHIgM22-treated TMEV mice compared to vehicle-treated TMEV mice. The reduction in $\mathrm{T}_{2}$ hyperintensities in $\mathrm{rHIgM} 22$-treated TMEV mice appeared to correspond with remyelination assessed using histology. ${ }^{38}$

\section{Cuprizone}

The cuprizone model involves ingestion of cuprizone, a copper chelator, in the animal's diet, which leads to demyelination in the CNS, particularly in the corpus callosum and superior cerebellar peduncles. ${ }^{39,40}$ Exposure to cuprizone 
for six weeks followed by a return to a normal diet for six weeks results in demyelination followed by spontaneous remyelination - this form of the disease is referred to as acute demyelination. If the cuprizone diet is continued for a full 12 weeks, chronic demyelination takes place..$^{40}$

\section{Assessing the effectiveness of potential therapeutics using MRI}

Several potential therapeutic agents have been evaluated in the cuprizone model using MRI, with the majority of these studies tracking white matter changes. Quetiapine is an antipsychotic agent that acts as an antagonist of the $\alpha 2$ adrenoceptor and the 5HT2A serotonin receptor. ${ }^{41}$ Quetiapine may have potential as a neuroregenerative agent for MS by stimulating proliferation and maturation of oligodendrocytes, and as an immunomodulator by inhibiting activated microglia and astrocytes. ${ }^{42}$ In the cuprizone model, use of quetiapine reduced pathological changes in white matter; specifically, fractional anisotropy was higher in quetiapine-treated cuprizone mice compared to vehicletreated cuprizone mice, ${ }^{43}$ indicative of greater preservation of tissue integrity in treated mice.

Olesoxime, a potential neuroregenerative agent, was applied in the cuprizone model, where it was observed that $\mathrm{T}_{2}$ signal intensity values in the corpus callosum of treated rats were closer to $T_{2}$ signal intensity values in controls than untreated cuprizone rats. ${ }^{44}$

In another cuprizone study, treatment with triiodothyronine $\left(\mathrm{T}_{3}\right)$ hormone led to radial diffusivity values approaching values seen in normal controls in parallel with improvements in remyelination in the genu and splenium of the corpus callosum, and in the cerebellum. ${ }^{45}$ Female sex hormones, namely estrogen and progesterone, have been applied in the cuprizone model where it was observed that $\mathrm{T}_{2}$ hyperintensity in the corpus callosum was reduced as was ventricle swelling in cuprizone mice treated with estrogen and progesterone compared to untreated cuprizone mice. ${ }^{46}$

Another cuprizone study compared salmon, cod liver oil and soybean oil diets in cuprizone mice, finding that mice on a salmon diet had significantly lower mean volumes of $\mathrm{T}_{2}$ hyperintensity than those receiving other diets. Histology showed reduced demyelination in mice on a salmon diet. These results are suggestive of a possible protective role of a fish-rich diet. ${ }^{47}$

One study investigated the effects of erythropoietin, which is considered a neuroprotective and neuroregenerative agent, on ventricular enlargement, finding that cuprizone mice treated with erythropoietin had reduced ventricular enlargement, assessed using $T_{1}$ mapping. ${ }^{48}$

\section{Lysolecithin}

The lysolecithin model is largely a demyelinating model with little lymphocytic inflammation; however, there is a significant representation of activated macrophages/microglia.
The model uses a focal injection of lysophosphatidylcholine, an activator of phospholipase A2, into a specific area in the $\mathrm{CNS}$, creating a demyelinating lesion at the site of injection. ${ }^{35}$ This model follows a predictable course of demyelination and subsequent remyelination that lasts approximately one month from beginning to end. The lysolecithin injection has been applied to the dorsal and ventral columns of the spinal cord, as well as to the corpus callosum and internal capsule.

\section{Assessing the effectiveness of treatment using MRI}

In contrast to some models described earlier, potential therapeutics have not been tested in this model with MRI extensively. One study used olesoxime in the lysolecithin model, finding that olesoxime reduced lesion size as measured using $\mathrm{T}_{2 .}{ }^{49}$

\section{Future directions: how to improve integration of MRI into animal model studies for assessment of potential therapeutics for MS}

Incorporating MRI into preclinical studies of therapeutics may aid in improving the design of clinical trials, since, as mentioned, MRI is commonly used as an outcome measure in phase II and III clinical trials. ${ }^{1}$ By including MRI when testing novel therapeutics in animal models of MS, changes in MRI metrics can be monitored and correlated with specific types of tissue responses (e.g. remyelination and/or reduction in inflammation). Such studies have been conducted while clinical trials have been taking place or before clinical trials even began, as is the case using natalizumab, ${ }^{18}$ fingolimod ${ }^{21}$ and anti-LINGO-1 in EAE. ${ }^{23}$ Ideally, studies testing potential MS drugs in animal models with MRI should be conducted prior to entering phase I clinical trials so that effects of the drug on MRI measures are known prior to being applied in the human MS population; such knowledge may provide a basis for the expected MRI outcomes in clinical trials. MRI methods for detecting BBB breakdown, inflammation, demyelination, and edema have all been tested in animal models, and are assumed to be similar for patients.

One obvious advantage of incorporating MRI into studies testing potential therapeutics for MS is the ability to image before applying the therapy to establish baseline measures. Furthermore, the effectiveness of potential agents can be assessed over a long period of time without having to sacrifice animals in the middle of the experiment. This reduces animal numbers as MRI enables for tracking changes over time, whereas when using histology only, a certain number of animals must be sacrificed at each time point. It also improves statistical analysis as one can undertake within-animal comparisons. 


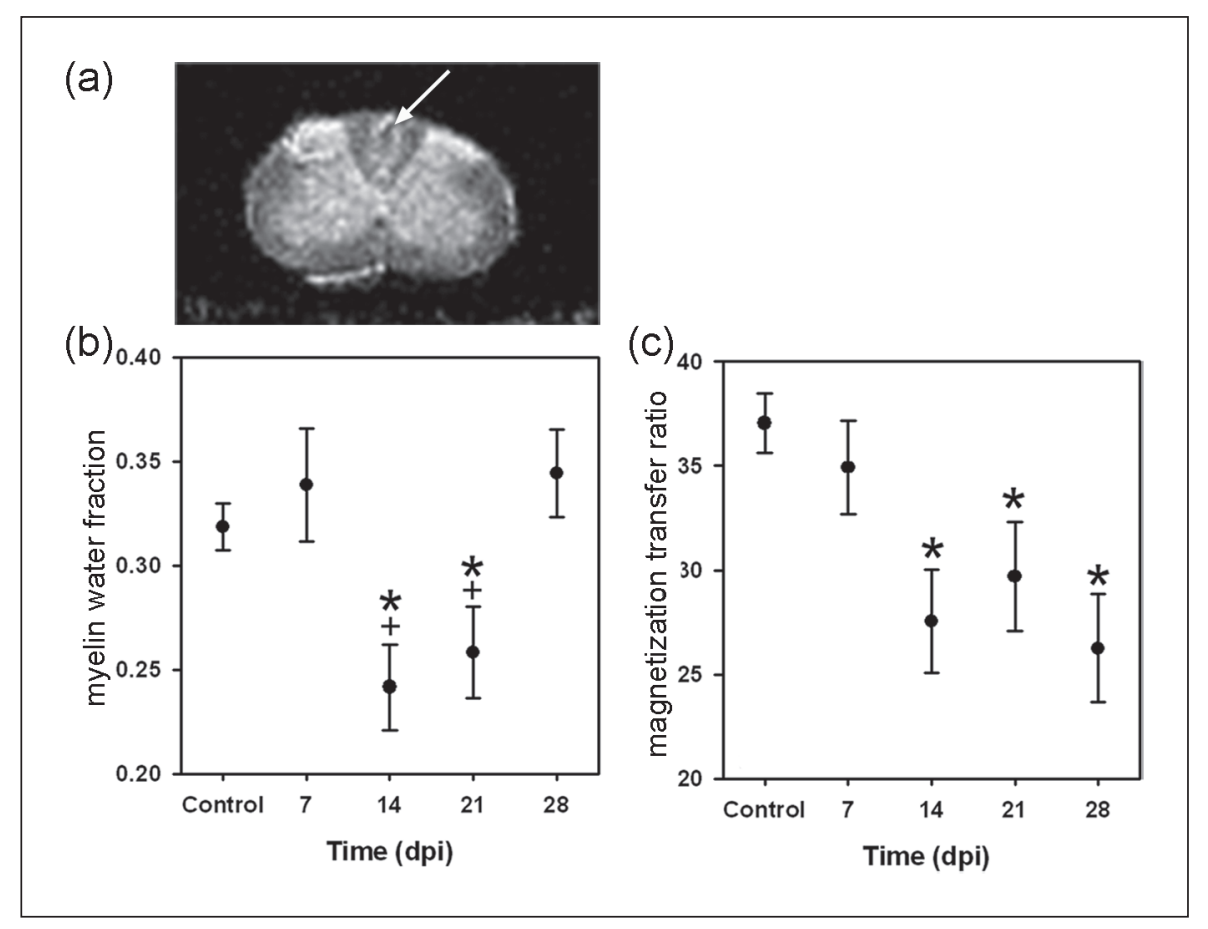

Figure 4. Myelin water imaging and magnetization transfer imaging can be used to follow lysolecithin-induced injury over a time course. (a) shows an ex vivo MRI of mouse spinal cord with a lysolecithin-induced demyelinating lesion (white arrow). (b) shows myelin water fraction values obtained from myelin water imaging (mean \pm SEM), where these values were significantly lower in lysolecithintreated mice than control mice at I4 and 21 days post-injection, but not at seven or 28 days. These changes are consistent with a demyelinating injury. (c) shows MTR values for the time course study, where MTR values were significantly lower in lysolecithin-treated mice than control mice at 14,21 and 28 days post-injection. These decreased values are indicative of reduced tissue integrity, and these values do not return to normal, unlike myelin water fraction values. ${ }^{*} p<0.05$ (Adapted from McCreary et al., 2009, Neuroimage ${ }^{50}$ ). MRI: magnetic resonance imaging; MTR: magnetization transfer ratio.

Each animal model has specific advantages for investigating potential treatments for MS using MRI. For example, the lysolecithin model may be an excellent model to test remyelinating agents that alter oligodendrocytes in the absence of destruction by lymphocytes. This model could be utilized to test agents using MRI methods sensitive to myelin such as DTI, MTI and myelin water imaging. The lysolecithin model has the additional advantage that one knows exactly where the lesion is, given that it is induced at a precise CNS location by the investigator. MTI and myelin water imaging have been used to track the lysolecithin lesion previously in the absence of drug treatment (Figure 4). ${ }^{50}$

The EAE model, having a large inflammatory component, is a good model to test the impact of anti-inflammatory agents in conjunction with MRI methods sensitive to inflammation, such as imaging with USPIOs. Although the typically utilized EAE model is useful, lesion location can be difficult to predict. Focal EAE models provide a means to follow a focal inflammatory, demyelinating lesion in a known location, over time, for treatment response, using MRI.

The cuprizone model may be useful to examine strategies that recover mitochondrial function or disruption in energy metabolism by incorporating perfusion and spectroscopy, which are sensitive to changes in metabolic patterns.

The TMEV model confers the advantage of testing therapeutic agents in the presence of a virally induced disease where demyelination is not due to direct viral cytopathic effects, but is instead due to a secondary immune response. ${ }^{34}$ Furthermore, hypointense areas have been observed in deep gray matter structures in the TMEV model ${ }^{51}$ and in $\mathrm{MS}^{52}$ using $\mathrm{T}_{2}$. Although there is no direct evidence that the underlying pathology is similar in these lesions, the similar phenotype on MRI is interesting, and the fact that $\mathrm{T}_{2}$ changes can be quantified means that response to treatments could be tested.

Some MRI methods that would be valuable, but that have not been used yet to evaluate potential therapeutics in animal models of MS include myelin water imaging, imaging with gadofluorine (Gf) M, magnetic resonance spectroscopy and susceptibility weighted imaging. Myelin water imaging, as the name suggests, is sensitive to myelin, and is based on the principle that the short component in $\mathrm{T}_{2}$ is due to water trapped between the myelin lipid bilayer. ${ }^{53,54}$ In instances where myelin is damaged or lost, myelin water content is reduced.$^{54}$ Imaging with $\mathrm{Gf}$ can be used to assess 
BBB breakdown, which has been shown to be more sensitive than Gd. ${ }^{55}$ Gf gives bright contrast on $\mathrm{T}_{1}$-weighted MRI and binds strongly to components of the extracellular matrix, including collagen and proteoglycans. ${ }^{56}$ Magnetic resonance spectroscopy would be useful for investigating metabolic alterations. Susceptibility weighted imaging, with its enhanced sensitivity to iron, ${ }^{57}$ would be useful for cell tracking and for investigating vascular alterations.

The necessity of incorporating MRI into studies testing potential therapeutic agents for MS at the preclinical stage cannot be understated. This will enable us to understand how potential therapeutics affect MRI metrics prior to entering clinical trials. Also, MRI metrics being assessed with potential therapies at the preclinical stage may inform on which of these MRI metrics would be useful to incorporate into clinical trials. With the many benefits that may be gained from incorporating MRI into studies assessing potential MS therapeutics in animal models, it should be made a priority in future studies.

\section{Conflict of interest}

The authors have no conflict of interest to report.

\section{Funding}

This work was supported by the Canadian Institutes of Health Research, the Natural Sciences and Engineering Research Council of Canada, Alberta Innovates - Health Solutions, the Multiple Sclerosis Society of Canada, and the Alberta endMS Regional Research and Training Centre of the endMS Research and Training Network.

\section{References}

1. Filippi M and Rocca MA. MR imaging of multiple sclerosis. Radiology 2011; 259: 659-681.

2. Stoll $\mathrm{G}$ and Bendszus M. New approaches to neuroimaging of central nervous system inflammation. Curr Opin Neurol 2010; 23: 282-286.

3. Dousset V, Brochet B, Deloire MS, et al. MR imaging of relapsing multiple sclerosis patients using ultra-small-particle iron oxide and compared with gadolinium. AJNR Am J Neuroradiol 2006; 27: 1000-1005.

4. Vellinga MM, Oude Engberink RD, Seewann A, et al. Pluriformity of inflammation in multiple sclerosis shown by ultra-small iron oxide particle enhancement. Brain 2008; 131: 800-807.

5. Lu M, Cohen MH, Rieves D, et al. FDA report: Ferumoxytol for intravenous iron therapy in adult patients with chronic kidney disease. Am J Hematol 2010; 85: 315-319.

6. Wolff SD and Balaban RS. Magnetization transfer contrast (MTC) and tissue water proton relaxation in vivo. Magn Reson Med 1989; 10: 135-144.

7. Filippi $M$ and Rovaris M. Magnetisation transfer imaging in multiple sclerosis. J Neurovirol 2000; 6 (Suppl 2): S115-S120.

8. Song SK, Sun SW, Ju WK, et al. Diffusion tensor imaging detects and differentiates axon and myelin degeneration in mouse optic nerve after retinal ischemia. Neuroimage 2003; 20: $1714-1722$.
9. Petrella JR and Provenzale JM. MR perfusion imaging of the brain: Techniques and applications. AJR Am J Roentgenol 2000; 175: 207-219.

10. Steinman L and Zamvil SS. How to successfully apply animal studies in experimental allergic encephalomyelitis to research on multiple sclerosis. Ann Neurol 2006; 60: 12-21.

11. Li DK, Li MJ, Traboulsee A, et al. The use of MRI as an outcome measure in clinical trials. Adv Neurol 2006; 98: 203-226.

12. Aharoni R, Sasson E, Blumenfeld-Katzir T, et al. Magnetic resonance imaging characterization of different experimental autoimmune encephalomyelitis models and the therapeutic effect of glatiramer acetate. Exp Neurol 2013; 240: 130-144.

13. Keough MB and Yong VW. Remyelination therapy for multiple sclerosis. Neurotherapeutics 2013; 10: 44-54.

14. Yong VW. Differential mechanisms of action of interferon-beta and glatiramer acetate in MS. Neurology 2002; 59: 802-808.

15. Zetterstrom M, Lundkvist J, Malinowsky D, et al. Interleukin-1-mediated febrile responses in mice and interleukin-1 beta activation of NFkappaB in mouse primary astrocytes, involves the interleukin-1 receptor accessory protein. Eur Cytokine Netw 1998; 9: 131-138.

16. Serres S, Bristow C, de Pablos RM, et al. Magnetic resonance imaging reveals therapeutic effects of interferon-beta on cytokine-induced reactivation of rat model of multiple sclerosis. $J$ Cereb Blood Flow Metab 2013; 33: 744-753.

17. Yednock TA, Cannon C, Fritz LC, et al. Prevention of experimental autoimmune encephalomyelitis by antibodies against alpha 4 beta 1 integrin. Nature 1992; 356: 63-66.

18. Deloire MS, Touil T, Brochet B, et al. Macrophage brain infiltration in experimental autoimmune encephalomyelitis is not completely compromised by suppressed T-cell invasion: In vivo magnetic resonance imaging illustration in effective antiVLA-4 antibody treatment. Mult Scler 2004; 10: 540-548.

19. Piraino PS, Yednock TA, Freedman SB, et al. Suppression of acute experimental allergic encephalomyelitis with a small molecule inhibitor of alpha4 integrin. Mult Scler 2005; 11: 683-690.

20. Aktas O, Kury P, Kieseier B, et al. Fingolimod is a potential novel therapy for multiple sclerosis. Nat Rev Neurol 2010; 6: 373-382.

21. Rausch M, Hiestand P, Foster CA, et al. Predictability of FTY720 efficacy in experimental autoimmune encephalomyelitis by in vivo macrophage tracking: Clinical implications for ultrasmall superparamagnetic iron oxide-enhanced magnetic resonance imaging. J Magn Reson Imaging 2004; 20: 16-24.

22. Wang X, Brieland JK, Kim JH, et al. Diffusion tensor imaging detects treatment effects of FTY720 in experimental autoimmune encephalomyelitis mice. NMR Biomed. Epub ahead of print 13 August 2013.

23. Mi S, Hu B, Hahm K, et al. LINGO-1 antagonist promotes spinal cord remyelination and axonal integrity in MOG-induced experimental autoimmune encephalomyelitis. Nat Med 2007; 13: $1228-1233$.

24. Song SK, Yoshino J, Le TQ, et al. Demyelination increases radial diffusivity in corpus callosum of mouse brain. Neuroimage 2005; 26: 132-140.

25. Yong VW, Wells J, Giuliani F, et al. The promise of minocycline in neurology. Lancet Neurol 2004; 3: 744-751.

26. Chen X, Hu X, Zou Y, et al. Combined treatment with minocycline and prednisone attenuates experimental autoimmune 
encephalomyelitis in C57 BL/6 mice. J Neuroimmunol 2009; 210: 22-29.

27. Jeong EA, Jeon BT, Shin HJ, et al. Ketogenic diet-induced peroxisome proliferator-activated receptor-gamma activation decreases neuroinflammation in the mouse hippocampus after kainic acid-induced seizures. Exp Neurol 2011; 232: 195-202.

28. Sullivan PG, Rippy NA, Dorenbos K, et al. The ketogenic diet increases mitochondrial uncoupling protein levels and activity. Ann Neurol 2004; 55: 576-580.

29. Kim do Y, Hao J, Liu R, et al. Inflammation-mediated memory dysfunction and effects of a ketogenic diet in a murine model of multiple sclerosis. PLoS One 2012; 7: e35476.

30. Berkovich R. Treatment of acute relapses in multiple sclerosis. Neurotherapeutics 2013; 10: 97-105.

31. Duckers HJ, Muller HJ, Verhaagen J, et al. Longitudinal in vivo magnetic resonance imaging studies in experimental allergic encephalomyelitis: Effect of a neurotrophic treatment on cortical lesion development. Neuroscience 1997; 77: 1163-1173.

32. Namer IJ, Steibel J, Poulet P, et al. Hyperbaric oxygen treatment in acute experimental allergic encephalomyelitis. Contribution of magnetic resonance imaging study. Neuroimage 1994; 1: 308-312.

33. Bennett $M$ and Heard R. Hyperbaric oxygen therapy for multiple sclerosis. CNS Neurosci Ther 2010; 16: 115-124.

34. Dal Canto MC, Kim BS, Miller SD, et al. Theiler's murine encephalomyelitis virus (TMEV)-induced demyelination: A model for human multiple sclerosis. Methods 1996; 10: 453461.

35. Denic A, Johnson AJ, Bieber AJ, et al. The relevance of animal models in multiple sclerosis research. Pathophysiology 2011; 18: 21-29.

36. Ciric B, Van Keulen V, Paz Soldan M, et al. Antibody-mediated remyelination operates through mechanism independent of immunomodulation. J Neuroimmunol 2004; 146: 153-161.

37. Mitsunaga Y, Ciric B, Van Keulen V, et al. Direct evidence that a human antibody derived from patient serum can promote myelin repair in a mouse model of chronic-progressive demyelinating disease. FASEB J 2002; 16: 1325-1327.

38. Pirko I, Ciric B, Gamez J, et al. A human antibody that promotes remyelination enters the CNS and decreases lesion load as detected by T2-weighted spinal cord MRI in a virusinduced murine model of MS. FASEB $J$ 2004; 18: 15771579 .

39. Blakemore WF. Demyelination of the superior cerebellar peduncle in the mouse induced by cuprizone. J Neurol Sci 1973; 20: 63-72.

40. Matsushima GK and Morell P. The neurotoxicant, cuprizone, as a model to study demyelination and remyelination in the central nervous system. Brain Pathol 2001; 11: 107-116.

41. Prieto E, Micó JA, Meana JJ, et al. Neurobiological bases of quetiapine antidepresant effect in the bipolar disorder [in English and Spanish]. Actas Esp Psiquiatr 2010; 38: 22-32.
42. Zhornitsky S, Wee Yong V, Koch MW, et al. Quetiapine fumarate for the treatment of multiple sclerosis: Focus on myelin repair. CNS Neurosci Ther 2013; 19: 737-744.

43. Chandran P, Upadhyay J, Markosyan S, et al. Magnetic resonance imaging and histological evidence for the blockade of cuprizone-induced demyelination in C57BL/6 mice. Neuroscience 2012; 202: 446-453.

44. Li Y, Zhang Y, Han W, et al. TRO19622 promotes myelin repair in a rat model of demyelination. Int J Neurosci 2013; 123: 810-822.

45. Harsan LA, Steibel J, Zaremba A, et al. Recovery from chronic demyelination by thyroid hormone therapy: Myelinogenesis induction and assessment by diffusion tensor magnetic resonance imaging. J Neurosci 2008; 28: 14189-14201.

46. Acs P, Kipp M, Norkute A, et al. 17beta-estradiol and progesterone prevent cuprizone provoked demyelination of corpus callosum in male mice. Glia 2009; 57: 807-814.

47. Torkildsen O, Brunborg LA, Thorsen F, et al. Effects of dietary intervention on MRI activity, de- and remyelination in the cuprizone model for demyelination. Exp Neurol 2009; 215: $160-166$.

48. Hagemeyer N, Boretius S, Ott C, et al. Erythropoietin attenuates neurological and histological consequences of toxic demyelination in mice. Mol Med 2012; 18: 628-635.

49. Magalon K, Zimmer C, Cayre M, et al. Olesoxime accelerates myelination and promotes repair in models of demyelination. Ann Neurol 2012; 71: 213-226.

50. McCreary CR, Bjarnason TA, Skihar V, et al. Multiexponential $\mathrm{T}_{2}$ and magnetization transfer MRI of demyelination and remyelination in murine spinal cord. Neuroimage 2009; 45: 1173-1182.

51. Pirko I, Johnson AJ, Lohrey AK, et al. Deep gray matter $T_{2}$ hypointensity correlates with disability in a murine model of MS. J Neurol Sci 2009; 282: 34-38.

52. Bakshi R, Benedict RH, Bermel RA, et al. T2 hypointensity in the deep gray matter of patients with multiple sclerosis: A quantitative magnetic resonance imaging study. Arch Neurol 2002; 59: 62-68.

53. Laule C, Vavasour IM, Leung E, et al. Pathological basis of diffusely abnormal white matter: Insights from magnetic resonance imaging and histology. Mult Scler 2011; 17: 144-150.

54. MacKay A, Whittall K, Adler J, et al. In vivo visualization of myelin water in brain by magnetic resonance. Magn Reson Med 1994; 31: 673-677.

55. Stoll G, Kleinschnitz C, Meuth SG, et al. Transient widespread blood-brain barrier alterations after cerebral photothrombosis as revealed by gadofluorine M-enhanced magnetic resonance imaging. J Cereb Blood Flow Metab 2009; 29: 331-341.

56. Meding J, Urich M, Licha K, et al. Magnetic resonance imaging of atherosclerosis by targeting extracellular matrix deposition with Gadofluorine M. Contrast Media Mol Imaging 2007; 2: 120-129.

57. Haacke EM, Xu Y, Cheng YC, et al. Susceptibility weighted imaging (SWI). Magn Reson Med 2004; 52: 612-618. 